\title{
Spectroscopy of yttrium dimers in argon matrices
}

\author{
Li Fang, Xiaoyu Chen, Xiaole Shen, Yifei Liu, \\ D. M. Lindsay, and John R. Lombardi \\ Department of Chemistry and Center for Analysis of Structures and Interfaces (CASI) \\ The City College of New York (CCNY), New York, NY10031, USA \\ E-mail: lombardi@scisun.sci.ccny.cuny.edu \\ Received February 5, 2000
}

\begin{abstract}
The absorption and resonance Raman spectra of yttrium dimers $\left(\mathrm{Y}_{2}\right)$ in argon matrices have been measured for the first time. The absorption spectrum (scattering depletion spectrum SDS) shows a weak, broad transition centered near $485 \mathrm{~nm}$. Resonance Raman spectra obtained by exciting into this absorption band with several visible laser lines $(465.5-496.5 \mathrm{~nm})$ give a single, sharp progression with up to ten Stokes transitions. These data give $\omega_{e}=184.4(4) \mathrm{cm}^{-1}$, with $\omega_{e} x_{e}=0.30(3) \mathrm{cm}^{-1}$, leading to a spectroscopic dissociation energy of $D_{e}=3.5(4) \mathrm{eV}$. Comparison of our results with several ab initio calculations adds confirmation to the assignment of the ground state of $\mathrm{Y}_{2}$ to be ${ }^{1} \Sigma_{g}^{+}$state.
\end{abstract}

PACS: 78.30.Hv, 71.55.Ht

\section{Introduction}

In the past two decades, research on transitionmetal clusters has attracted a number of theoretical and experimental scientists [1]. It is clear that an understanding of the multiple-metal bonding often observed in the ground states of transition metal dimers depends on accurate determination of crucial structural parameters such as vibrational frequencies, force constants, dissociation energies, etc. Work has been carried out in this regard by this and other laboratories on several second row transition metal dimers $\left(\mathrm{Zr}_{2}\right.$ [2], $\mathrm{Nb}_{2}$ [3], $\mathrm{Mo}_{2}$ [4], $\mathrm{Ru}_{2}$ [5], $\mathrm{Rh}_{2}$ [6], $\mathrm{Pd}_{2}$ [7], and $\mathrm{Ag}_{2}$ [8]). However, relatively little work has been carried out on the dimer of yttrium. In present paper we report the first matrix isolation optical absorption and resonance Raman spectra of $\mathrm{Y}_{2}$. Previous work on the atomic spectrum has been reported by Klotzbuecher and Reva [9].

\section{Experiment}

The City College of New York metal cluster deposition source has been described in previous publications $[2,3,10]$. Briefly, an intense (typically $15 \mathrm{~mA}$ at $25 \mathrm{keV}$ ) argon ion beam from a CORDIS ion source sputters on a water cooled yttrium target (Alfa, 99.9\%) maintained at $300 \mathrm{~V}$. Secondary ions are extracted with a modified Colutron model 200$B$ lens system and then mass selected using a Wien filter (Colutron 600-B) in conjunction with an approximately $175 \mathrm{~mm}$ free drift distance and a $6.5 \mathrm{~mm}$ diameter aperture. The mass resolution is 6-7, enough to discriminate against possible oxide contaminants, as well as provide unambiguous filtering and metal clusters or atoms. After mass selection, the ion beam is bent by $10^{\circ}$ using two electric plates to eliminate neutrals and then guided and focused to the deposition region by two einzellike lenses.

Samples of $\mathrm{Y}_{2}$ are obtained by mass selection after sputtering a metal target with high energy $\mathrm{Ar}$ ions. This ensures discrimination against spectral interference from atoms and higher clusters of $\mathrm{Y}$, as well as various oxides of $\mathrm{Y}$. We observe a weak, broad optical transition near $485 \mathrm{~nm}$. Exciting with $\mathrm{Ar}^{+}$laser radiation in this region enables us to obtain a sharp resonance Raman spectrum with a long progression of overtones.

Yttrium dimer ions were codeposited with $\mathrm{Ar}$ and electrons (generated from a heated tungsten filament) on a polished $\mathrm{CaF}_{2}$ substrate $(\sim 14 \mathrm{~K})$. Matrices were grown at $\sim 5 \mu \mathrm{m} / \mathrm{h}$ with an $\mathrm{Ar}$ : metal dilution ratio of approximately $10^{4}: 1$. The deposition region was surrounded by a «Faraday Cage» whose potential with respect to the sputtering target controls the kinetic energy ( $10 \mathrm{eV}$ in this experiment) of deposited ions. Ion currents under «soft landing» conditions were approximately: $\mathrm{Y}^{+}$ $(250 \mathrm{nA}), \mathrm{Y}_{2}^{+}(25 \mathrm{nA})$. 


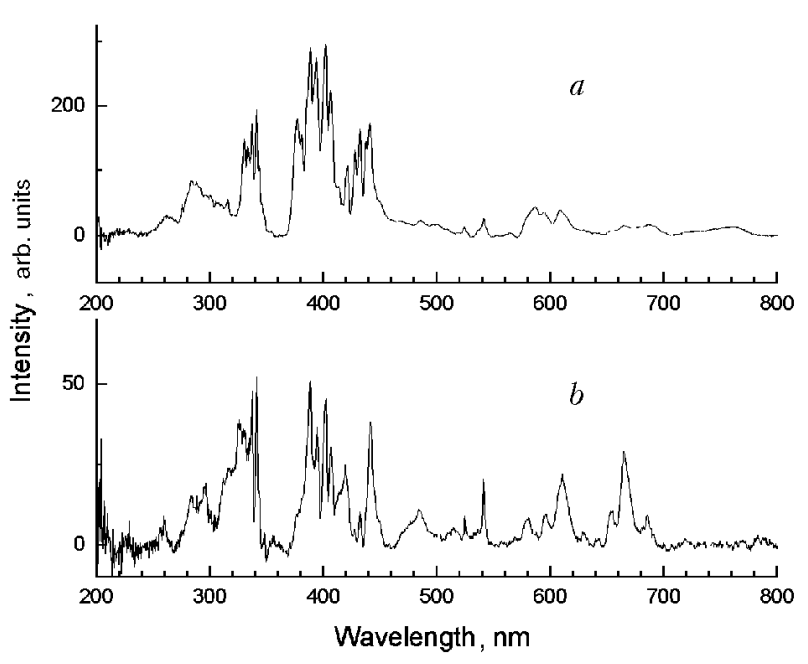

Fig. 1. Scattering depletion spectra of atom (a) and of yttrium dimer $(b)$.

Under similar conditions, we deposited the yttrium atom in an argon matrix. By comparing the intensity of known atomic excitation features in a dimer deposition with those obtained from deposition of atom under similar conditions, the fragmentation ratio of dimers is estimated to be $10 \%$.

Matrix samples were interrogated in situ using both absorption and Raman spectroscopy. As previous described $[2,3,10]$, for the absorption measurements, both deuterium and tungsten lamps were employed as excitation light sources, dispersed by a single $1 / 4 \mathrm{~m}$ monochromator, reflected by a plane mirror (controlled by a stepping motor), which allows the light to be scanned across the $8 \mathrm{~mm}$ wide sample. The absorption measurements were made by collecting the light scattered at $90^{\circ}$ to that incident, a technique termed «Scattering Depletion Spectroscopy (SDS)» [3].

After obtaining absorption spectra, the Raman spectra were measured by exciting the sample with appropriate laser wavelength within the absorption region. In this experiment on $\mathrm{Y}_{2}$, the visible output $(457.9-514.5 \mathrm{~nm}$ ) of an argon ion laser (Spectra Physics model 2045) was employed. The scattered light was collected at $90^{\circ}$ and focused into a Triplemate Spectrometer (Spex 1877E, $0.6 \mathrm{~m}$ ). The scattered light was detected with a liquid nitrogen

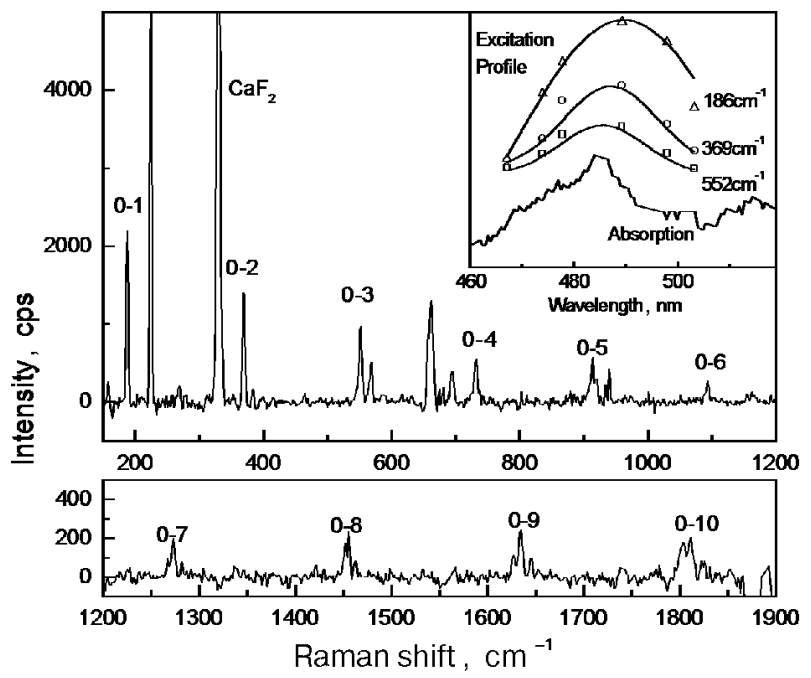

Fig. 2. Typical Raman spectra for $\mathrm{Y}_{2}$ in argon matrix.

cooled CCD system (Spectrum One + CCD30 + DM3000R Software). The resolution of the detection system for the $\mathrm{Y}_{2}$ experiment was set about $2 \mathrm{~cm}^{-1}$ (at $500 \mathrm{~nm}$ ). The Raman shifts of $\mathrm{Y}_{2}$ were calibrated using the $\mathrm{CaF}_{2}$ (substrate at $16 \mathrm{~K}$ ) line at $327 \mathrm{~cm}^{-1}$.

\section{Spectra and analysis}

Figure 1 shows the scattering depletion spectrum (SDS) of the atom $(a)$, which is essentially the same as Klotzbuecher's result [9], and of the dimer of yttrium $(b)$.

Figure 2 shows a typical Raman spectrum (excited with $488 \mathrm{~nm}$ ) for $\mathrm{Y}_{2}$ in argon matrix $(14 \mathrm{~K}$, dimer content $\sim 75 \mathrm{nA} / \mathrm{h}$ ) and, as an inset, a portion of the absorption spectrum (SDS) of the same sample compared with several excitation profiles.

The SDS shows a broad, weak band centered at $485 \mathrm{~nm}$, and closely parallels the excitation profile, which further confirms its assignment to the yttrium dimer.

Resonance Raman spectra of $Y_{2}$ were obtained at five different argon ion laser emissions in the visible region $(465.5-496.5 \mathrm{~nm})$. Up to ten Stokes transitions were observed and the average value of these

Table 1

Raman frequency shifts for yttrium dimers in argon matrices

\begin{tabular}{|c|c|c|c|c|c|c|c|c|c|c|}
\hline$v^{\prime \prime}$ & 1 & 2 & 3 & 4 & 5 & 6 & 7 & 8 & 9 & 10 \\
\hline Raman shift, $\mathrm{cm}^{-1}$ & 183.5 & 366.1 & 548.8 & 730.7 & 912.5 & 1093.3 & 1273.6 & 1454.3 & 1632.3 & 1810.1 \\
\hline Std. Dev. & 0.6 & 0.4 & 0.7 & 0.7 & 0.7 & 0.7 & 0.4 & 0.6 & 1.0 & 1.3 \\
\hline
\end{tabular}


Of several previous publications on $\mathrm{Y}_{2}$ only one

measurements give the Raman shifts, which are listed in Table 1.

Analysis of these data by standard techniques [11] (linear fit of $\Delta G_{v+1 / 2}$ vs. v) gives $\omega_{e}=$ $=184.4(4) \mathrm{cm}^{-1}$, with $\omega_{e} x_{e}=0.30(3) \mathrm{cm}^{-1}$, leading to a spectroscopic dissociation energy of $D_{e}=3.5(4) \mathrm{eV}$, and the force constant $k_{e}=$ $=0.90$ (1) mdyne $/ \AA$. Attempts to obtain higher anharmonic corrections $\left(\omega_{e} y_{e}\right)$ failed to improve the standard deviation, so it may be safely inferred that such corrections are negligible. Our observed vibrational frequency is essentially the same as that of Yang et al. [12] $\omega_{e}=185(2)$ obtained by ZEKE spectroscopy.

Note that several of the observed Raman lines are accompanied by one or more weaker satellite lines. Since there are no isotopes that need to be considered, it is most likely that they are due to site effects in the Ar matrix. Similar effects have been observed in other dimer spectra [3]. We have used only the most intense line in each group for our analysis.

\section{Discussion}

It is of interest to compare our measured value of the force constant $k_{e}$ for $\mathrm{Y}_{2}$ with those of other members of the second row transition metal dimers. These are given in Table 2 .

Table 2

Ground state vibrational frequencies $\omega_{e}$ and force constants $k_{e}$ for several second row transition metal dimers

\begin{tabular}{c|c|c|c|c}
\hline \hline & $\mathrm{Y}_{2}$ (this work) & $\mathrm{Zr}_{2}(2)$ & $\mathrm{Nb}_{2}(3)$ & $\mathrm{Mo}_{2}(4)$ \\
\hline \hline$\omega_{e}, \mathrm{~cm}^{-1}$ & 184.4 & 305.7 & 420.5 & 477.1 \\
$k_{e}$, mdyne $/ \AA$ & 0.90 & 2.51 & 4.84 & 6.43 \\
\hline \hline
\end{tabular}

As can be seen the force constants increase almost linearly from left to right. The ground state atomic configurations are (Y) $5 s^{2} 4 d^{1}$, ( $\mathrm{Zr}$ ) $5 s^{2} 4 d^{2}$, (Nb) $5 s^{1} 4 d^{4}$, (Mo) $5 s^{1} 4 d^{5}$. However, in transition metals, bonding is usually more favorable if at least one atom has an $s^{1}$ configuration, requiring some promotion energy. Thus, considering a configuration $5 s^{1} 4 d^{m}$ ( $\left.m=2-5\right)$, the force constant is proportional to the number of $d$ electrons involved in bonding. (A complementary, nearly linear decline in force constant was observed [5-7] in the series $\mathrm{Ru}_{2}, \mathrm{Rh}_{2}, \mathrm{Pd}_{2}$ indicating a configuration $5 s^{1} 4 d^{m}$ $(m=7-9))$. We can conclude that, at least for the early second row transition metal dimers, each $d$ electron available for bonding makes a nearly equal additional contribution to the bond order. is experimental, Verhaegan, Somoes, and Drowart [13] determined the bonding energy using the third law analysis of the high temperature Knudsen effusion mass spectrum. Their result was $D_{0}\left(\mathrm{Y}_{2}\right)=1.62 \pm 0,22 \mathrm{eV}$, in sharp disagreement with our spectroscopic value of $3.5 \pm 0.4 \mathrm{eV}$. The third law technique suffers from the requirement that $\omega_{e}$ and $r_{e}$ must be known, as well as the electronic partition function, and the results are often unreliable. However, the spectroscopic technique also has difficulties, in that a Morse potential is assumed to govern the nuclear motion. In transition metals this assumption is not always even close. This is caused by the fact that, where both $s-s$ and $d-d$ bonding is important, due to their disparate spatial extensions, $d$ orbitals often have considerably different dissociation ranges than $s$ orbitals. The best example of this is the case of $\mathrm{Cr}_{2}$ [14], where serious deviations from a Morse potential are observed and the spectroscopic value is misleading. However, in $\mathrm{Y}_{2}$ it is most likely that the bond order is small, perhaps near one. Thus, only one electron pair is involved in dissociation, and the above effect of different $s$ and $d$ bonding will not be important. We also have observed regular behavior all the way up to ninth harmonic $(n=10)$. We feel, therefore, that our value for $D_{e}$ is more likely to be correct.

The remaining work on $\mathrm{Y}_{2}$ has been theoretical. Walch and Bauschlicher [15] have carried out a complete active-space multiconfiguration-self-consistent field (CAS-MCSCF) calculation followed configuration-interaction (CI). Their results indicate that the ground state is ${ }^{5} \Sigma_{u}^{-}$ $\left(5 s \sigma_{g}^{2} 5 s \sigma_{u}^{1} 4 d \sigma_{g}^{1} 4 d \pi_{x u}^{1} 4 d \pi_{y u}^{1}\right)$ which stems from the $5 s^{2} 4 d^{1}+5 s^{1} 4 d^{2}$ atomic configurations. A nearby, low-lying state $\left(T_{c}=0.87 \mathrm{eV}\right)$ is the ${ }^{1} \Sigma_{g}^{+}$ $\left(5 s \sigma_{g}^{2} 4 d \pi_{x u}^{2} 4 d \pi_{y u}^{2}\right) \quad$ state arising from the $5 s^{1} 4 d^{2}+5 s^{1} 4 d^{2}$ configurations. In similar calculations in $\mathrm{Sc}_{2}$ this latter state lies considerably higher than that in $\mathrm{Y}_{2}$ which indicates larger contributions of $d$-electron bonding in the second row. Dai and Balasubramanian [16] have carried out similar complete active space self consistent field (CASSCF) calculations followed by multi-reference configuration interaction (MRSDCI) in which both single and double excitations are considered. Up to 2.6 million configurations are included in this calculation. Their results are quite similar to those of Walch and Bauschlicher (see Table 3). Comparison 
Theoretical and thermodynamic results for low state parameters of $\mathrm{Y}_{2}$ and spectroscopic data (this work)

\begin{tabular}{|c|c|c|c|c|c|c|}
\hline Reference & Method & Low state & $r_{e}, \mathrm{~A}$ & $\omega_{e}, \mathrm{~cm}^{-1}$ & $D_{e}$ or $D_{0}, \mathrm{eV}$ & $T_{e}, \mathrm{eV}$ \\
\hline D. G. Dai and & $\mathrm{CASSCF}+$ & ${ }^{5} \Sigma_{u}^{-}$ & 3.03 & 172 & 2.56 & 0.0 \\
\hline K. Balasubramanian [15] & MRSDCI & ${ }^{1} \Sigma_{g}^{+}$ & 2.76 & 180 & 3.09 & 0.55 \\
\hline \multirow{3}{*}{$\begin{array}{c}\text { S. P. Walch and } \\
\text { C. W. Bauschlicher, Jr. [14] }\end{array}$} & CI & ${ }^{5} \Sigma_{u}^{-}$ & 3.03 & 171 & 2.44 & 0.00 \\
\hline & CI & ${ }^{1} \Sigma_{q}^{+}$ & 2.74 & 206 & 2.93 & 0.87 \\
\hline & CASSCF & ${ }^{1} \Sigma_{g}^{+}$ & 2.73 & 205 & 1.74 & - \\
\hline $\begin{array}{c}\text { G. Verhaegan, S. Somoes, } \\
\text { and J. Drowart [12] }\end{array}$ & & & & & $1.62 \pm 0.22$ & \\
\hline This work & Isolated matrix & & & $184.4 \pm 0.4$ & $3.5 \pm 0.4$ & \\
\hline
\end{tabular}

of the SCF calculations both with and without CI contributions show that increasing CI lowers the relative energy of the higher state considerably (to $\left.T_{e}=0.55 \mathrm{eV}\right)$. In conjunction with the lack of observable ESR spectrum [17], Dai and Balasubramanian conclude that the ${ }^{1} \Sigma_{g}^{+}$state is most likely the ground state. Our Raman results (see Table 3) are consistent with this conclusion. The experimental value for $\omega_{e}$ of $184.4 \mathrm{~cm}^{-1}$ is quite close to that of $180 \mathrm{~cm}^{-1}$ in the CASSCF+MRSDCI calculation of Dai and Balasubramanian for the ${ }^{1} \Sigma_{g}^{+}$state. Similarly our $D_{e}=3.5(4) \mathrm{eV}$ compares favorably with their value of $3.09 \mathrm{eV}$ for the same state.

The theoretical results are listed and compared with this work in Table 3 .

Due to the rather large calculated differences in $r_{e}$ for the ${ }^{5} \Sigma_{u}^{-}$and ${ }^{1} \Sigma_{g}^{+}$states, the determination of an accurate experimental value for $r_{e}$ would be helpful to determine the nature of the ground state. Badger's [18] rule may be employed here on the fourth row metal diatomic molecules following Weisshaar's results [19] on the third row metal elements. Unfortunately, only a very few experimental data (listed in Table 4) have been reported until now, especially of the bond length $r_{e}$. The normal form of Badger's rule has the format: $k_{e}\left(r_{e}-d_{i j}\right)^{3}=C$, where $d_{i j}$ is a constant that is different depending one the row number of the periodical table in which each atom resides and $C$ may be taken as the same for all molecules $\left(C=1.86 \mathrm{mdyn} \cdot \AA^{2}\right.$ if $k_{e}$ is in mdyn $/ \AA$ and $r_{e}, d_{i j}$ in $\AA$ ). However, it is found that allowing $C$ to vary with periodic table row gives considerably better fits $[19,20]$. The $C$ value in Ref. 19 varies from 0.53 $(3,4)$ to $5.83(H, 3 T)$ according to the rows of the periodic table.

Table 4

Experiment data of the third row metal diatomic molecules

\begin{tabular}{c|c|c|c|c|c}
\hline \hline Molecule & State & $\omega_{e}, \mathrm{~cm}^{-1}$ & $r_{e}, \AA$ & $k_{e}^{-1 / 3},(\mathrm{mdyn} / \mathrm{A})^{-1 / 3}$ & Reference \\
\hline \hline $\mathrm{Mo}_{2}$ & $X^{1} \Sigma_{g}^{+}$ & 477.1 & 1.938 & 0.537 & {$[21]$} \\
$\mathrm{Mo}_{2}$ & $A^{1} \Sigma_{u}^{+}$ & 449 & 1.937 & 0.560 & {$[21]$} \\
$\mathrm{Ag}_{2}$ & $X\left(O_{g}^{+}\right)^{1} \Sigma_{g}^{+}$ & 192.4 & 2.531 & 1.095 & {$[1,22]$} \\
$\mathrm{Ag}_{2}$ & $A\left(O_{u}^{+}\right)^{1} \Sigma_{u}^{+}$ & 155.3 & 2.655 & 1.816 & {$[1,22]$} \\
$\mathrm{Sr}_{2}$ & $B^{1} \Pi_{u}$ & 80.4 & 3.85 & & {$[23]$} \\
\hline \hline
\end{tabular}




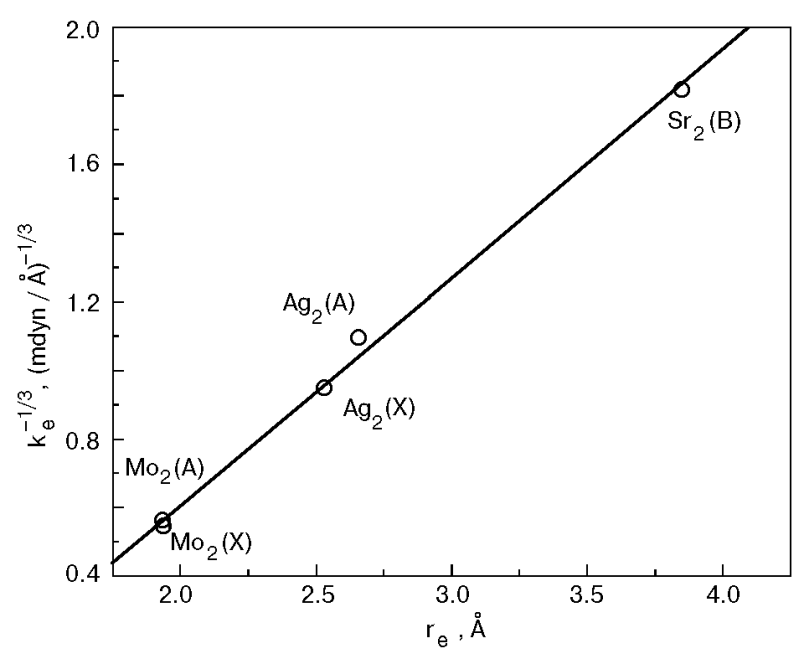

Fig. 3. Correlation between force constant $k_{e}$ and parameter $r_{e}$.

In order to determine the constant $d_{44}$ and $C$ of the forth row metal diatomic molecules, a linear least squares fit was employed to five pairs of experimentally determined $\omega_{e}, r_{e}$ (see Table 4 and Fig. 3), leading to $d_{44}^{e}=1.09 \AA$ and $C=$ $=3.41 \mathrm{mdyn} \cdot \AA^{2} \quad$ (linear correlation coefficient is 0.998). Using our experimental result $\omega_{e}=184.4 \mathrm{~cm}^{-1}$, the bond length of $\mathrm{Y}_{2}$ may be obtained as an interpolated point on this graph. It is found to be $2.65 \AA$, which is only slightly shorter than Dai's result $\left(r_{e}=2.76 \AA\right)$ for the ${ }^{1} \Sigma_{q}^{+}$state. For the ${ }^{5} \Sigma_{u}^{-}$state, his result is $3.03 \AA$. This lends additional support to the presumption that ${ }^{1} \Sigma_{g}^{+}$is very likely the ground state.

\section{Acknowledgments}

This work was supported by the National Science Foundation under Cooperative Agreement No. RII9353488 and Grant No. CHE-9412804 and by the City University of New York PSC-BHE Faculty Research Award Program. We are also indebted to Dr. Werner E. Klotzbuecher for his helpful discussion of the atomic spectra.
1. M. D. Morse, Chem. Rev. 86, 1049 (1986).

2. Z. Hu, Q. Zhou, J. R. Lombardi, D. M. Lindsay, in: Spectroscopy of Mass-Selected Zirconium Dimers in Argon, in Physics and Chemistry of Finite Systems: from Clusters to Crystals, P. Jena, S. N. Kahana, and B. K. Rao (eds.), Kluwer Academic, Dordrecht (1992).

3. Z. Hu, B. Shen, S. Deosaran, J. R. Lombardi, D. M. Lindsay, and W. Harbich, Proc. SPIE 1599, 65 (1991).

4. Y. M. Efremov, A. N. Samoilova, V. B. Kozhukhovsky, and L. B. Gurvich, J. Mol. Spectrosc. 73, 430 (1978).

5. H. Wang, Y. Liu, H. Haouri, R. Craig, J. R. Lombardi, and D. M. Lindsay, J. Chem. Phys. 106, 6534, (1997).

6. H. Wang, H. Haouri, R. Craig, Y. Liu, J. R. Lombardi, and D. M. Lindsay, J. Chem. Phys. 106, 2101 (1997).

7. J. Ho,. K. M. Ervin, K. L. Polak, M. K. Gilles, and W. C Lineberger, J. Chem. Phys. 95, 4845 (1991).

8. K. P. Huber and G. Herzberg, Constants of Diatomic Molecules, Van Norstrand, New York (1979).

9. E. W. Klotzbucher and I. D. Reva, in: XVII IUPAC Symp. on Photochemistry, Barcelona, Spain, 19-24.July (1998).

10. Z. Hu, B. Shen, S. Deosaran, J. R. Lombardi, D. M. Lindsay, and W. Harbich, J. Chem. Phys. 95, 2206 (1991).

11. G. Herzberg, Molecular Spectra and Molecular Structure, I. Spectra of Diatomic Molecules, D. Van Nostrand Company, Inc. New York (1950).

12. D. S. Yang, B. Simard, P. A. Hackett, A. Breces, and M. Z. Zgierski, Int. J. of Mass Spectrometry and Ion Processes 159, 65 (1996).

13. G. Verhaegan, S. Somoes, and J. Drowart, J. Chem. Phys. 40, 239 (1964).

14. S. M. Casey and D. G. Leopold, J. Phys. Chem. 97, 816 (1993).

15. S. P. Walch and C. W. Bauschlicher, C. W., Jr. in: Comparison of ab initio Quantum Chemistry with Experiment for Small Molecules, R. J. Bartlett (ed), D. Reidel: Dordrecht (1985).

16. D. G. Dai and K. Balasubramanian, J. Chem. Phys. 98, 7098 (1993)

17. L. B. Knight, Jr., R. W. Woodward, R. J. Van Zee, and W. Jr. Weltner, J. Chem. Phys. 79, 5820 (1983).

18. R. M. Badger, J. Chem. Phys. 2, 128 (1934); ibid. 3, 710 (1935).

19. J. C. Weisshaar, J. Chem. Phys. 90, 1429 (1989).

20. D. R. Herschbach and V. W. Laurie, J. Chem. Phys. 35, 458, (1961)

21. J. B. Hopkins, P. R. R. Langridge-Smith, M. D. Morse, and R. E. Smalley, J. Chem. Phys. 781627 (1983).

22.B. Simard and P. A. Hackett, Chem. Phys. Lett. 186, 4 (1991).

23. C. Bordas, M. Broyer, J. Chevaleyre, and Ph. Dugourd, Chem. Phys. Lett. 197, 562 (1992). 